\title{
Die Tibetische Rezeptur Padma Lax in der ärztlichen Praxis: Eine retrospektive Fallstudie bei Obstipation mit 174 Patienten
}

\author{
Bernhard Uehleke $^{\mathrm{a}}$ Simona Zahradnicek ${ }^{\mathrm{a}}$ Cecile Vennos $^{\mathrm{b}}$ \\ ${ }^{a}$ Institut für Naturheilkunde, Universitätsspital Zürich, \\ ${ }^{\text {b }}$ Padma AG, Hinwil, Schweiz
}

\section{Schlüsselwörter}

Padma Lax · Retrospektive Fallstudie - Obstipation . Tibetische Medizin . Phytotherapie

\section{Zusammenfassung}

Hintergrund: Das Tibetische Arzneimittel Padma Lax ist seit über 40 Jahren in der Schweiz bei Obstipation und Blähungen zugelassen. In dieser Studie wurden Daten zur Anwendung im Praxisumfeld und zur Wirksamkeit bei verschiedenen obstipationsbedingten Beschwerden sowie zu Sicherheit und Verträglichkeit erhoben und bewertet. Material und Methoden: In der Schweiz praktizierende Ärztinnen und Ärzte dokumentierten retrospektiv den Behandlungsverlauf bei chronischer Obstipation mittels eines eigens entwickelten Fragebogens. Neben der Stuhlfrequenz wurde unter anderem auch die Intensität von 12 spezifischen Verstopfungssymptomen erhoben. Ergebnisse: 17 teilnehmende Ärzte lieferten Daten von insgesamt 174 Patientenfällen, wobei rund $30 \%$ aus der gynäkologischen Praxis stammten. Als Obstipationsursachen wurden unter anderem Lifestyle-Faktoren und verschiedene Grunderkrankungen angegeben, z.B. neurologische oder hormonelle Störungen. Sicherheit und Verträglichkeit wurden als sehr gut beurteilt, es wurde nur 1 unerwünschtes Ereignis (eine Verschlechterung der Flatulenz) angegeben. Die Stuhlfrequenz stieg im Behandlungsverlauf deutlich, $97 \%$ erreichten eine Frequenz von $>2$ pro Woche. Durchschnittlich verringerten sich die Häufigkeit und die Intensität aller Symptome, mit Ausnahme des Symptoms weicher/dünner Stuhl, das sich bei ebenso vielen Patienten erhöhte wie erniedrigte. Die Gesamtintensität über alle Symptome sank von durchschnittlich 15,8 um $63 \%$ auf 5,8. Der Anteil der Patienten mit starken Beschwerden (Gesamtscore $>20$ ) sank von $20,1 \%$ auf $1,7 \%$, was in der Folge die Gruppe mit "kaum Beschwerden» (Gesamtscore $\leq 5$ ) von 1,2\% auf $57 \%$ ansteigen ließ. 86\% der Ärzte und der Patienten beurteilten die Wirksamkeit als gut. Schlussfolgerungen: Padma Lax wurde bei Verstopfung unterschiedlicher Ursache bei einem breiten Patientenspektrum ein gesetzt. Die Einschätzung der Wirksamkeit und Verträglichkeit war gut oder sehr gut. Trotz der Limitierung durch den retrospektiven Studientyp weisen die Daten darauf hin, dass Padma Lax bei chronischer Verstopfung unterschiedlicher Ursache, insbesondere auch in der gynäkologischen Praxis, bei geriatrischen Patienten und bei $\mathrm{Pa}-$ tienten mit neurogener Obstipation, eine wirksame und verträgliche Therapieoption darstellt.

Hinweis: Padma Lax (Swissmedic Nr. 35872) ist in der Schweiz auch unter dem Namen Padmed Laxan (Swissmedic Nr. 57478) erhältlich und kassenzulässig.

\section{Keywords}

Padma Lax · Retrospective case study · Constipation . Tibetan Medicine $\cdot$ Phytotherapy

\section{Summary}

The Tibetan Formula Padma Lax in Medical Practice: A Retrospective Case Series Study in Constipation Including 174 Patients

Background: The Tibetan Medicine Padma Lax has been approved in Switzerland for the treatment of constipation and bloating for over 40 years. In this study, data on the application in medical practice, as well as on effectiveness in various constipation-related symptoms and on safety and tolerability, were collected and assessed. Material and Methods: Physicians practicing in Switzerland documented retrospectively the course of treatment for chronic constipation, using an especially developed questionnaire. In addition to defecation frequency, data on the intensity of 12 specific symptoms of constipation were collected. Results: 17 participating physicians provided data from a total of 174 patient cases, about $30 \%$ of which originated from gynecological practices. Among others, lifestyle factors and various underlying diseases, e.g. neurological or hormonal disturbances, were indicated as causes of the constipation. Safety and tolerability were very good; only 1 adverse event (a worsening of flatulence) was reported. The frequency of defecation increased significantly during treatment and $97 \%$ reached a frequency of $>2$ per week. On average, the frequency and intensity of all symptoms decreased, with the exception of the symptom soft/loose stools, which increased in as many patients as it decreased. The overall intensity of all symptoms decreased from a mean of 15.8 by $63 \%$ to 5.8 . The proportion of patients with severe complaints (overall intensity score $>20$ ) decreased from $20.1 \%$ to $1.7 \%$, and as a result, the group with hardly any complaints (overall intensity score $\leq 5$ ) rose from $1.2 \%$ to $57 \%$. $86 \%$ of the physicians and the patients rated the efficacy as good. Conclusions: Padma Lax was used in constipation of different causes and in a broad spectrum of patients. Efficacy and safety were assessed as good or very good. In spite of the limitation of the retrospective study design, the data indicate that Padma Lax is an effective and well-tolerated treatment option for chronic constipation from different causes, especially also in gynecological practice, in geriatric patients, and in patients with neurogenic constipation.

\section{KARGER \\ Fax +497614520714 \\ Information@Karger.com}

www.karger.com
(C) 2013 S. Karger GmbH, Freiburg

$1661-4119 / 13 / 0208-0008 \$ 38.00 / 0$

Accessible online at:

www.karger.com/fok
Dr. med. vet. Cecile Vennos

Leiterin Regulatory Affairs

Unterfeldstrasse 1, 8340 Hinwil, Schweiz

c.vennos@padma.ch 


\section{Einleitung}

Das Tibetische Arzneimittel Padma Lax ${ }^{\circledR}$ ist seit über 40 Jahren in der Schweiz zugelassen (Swissmedic Nr. 35872) und ist damit eine der am längsten in Europa angewendeten Rezepturen aus einem asiatischen Medizinsystem. Das Präparat wird bei Obstipation und Blähungen eingesetzt und regt zusätzlich die Verdauungsfunktionen an. Es handelt sich um eine Rezeptur aus 15 pflanzlichen und mineralischen Komponenten.

Zur Anwendung im europäischen Kontext liegen einige wissenschaftliche Daten vor: Einzelfallberichte [1-3], 1 klinische Doppelblind-Studie bei Patienten mit obstipationsdominantem Reizdarmsyndrom (IBS) und 2 experimentelle Studien [4-6].

Ziel dieser retrospektiv angelegten Studie war es, unter Praxisbedingungen $\mathrm{zu}$ untersuchen, bei welchen Patienten und bei welchen Formen der Obstipation Ärztinnen und Ärzte in der Schweiz Padma Lax anwenden. Außerdem sollten Daten zur Dosierung und Therapiedauer sowie zur Sicherheit und Verträglichkeit erhoben werden. Weiter wurde die Wirksamkeit bei unterschiedlichen verstopfungsbedingten Symptomen evaluiert.

\section{Material und Methoden}

In der Schweiz praktizierende Ärzte aus den Fachgebieten Allgemeine und Innere Medizin, Chirurgie, Endokrinologie-Diabetologie, Gastroenterologie, Gynäkologie und Geburtshilfe, Urologie und Geriatrie wurden per Postversand eingeladen, retrospektiv Daten zur Behandlung von Obstipationspatienten mit Padma Lax zu erheben.

Die Daten wurden anhand eines eigens entwickelten Fragebogens erhoben. Dieser stützte sich auf die Symptome funktioneller Darmerkran kungen gemäß den Rome-III-Kriterien (insbesondere Abschnitt C3 «Functional Constipation») sowie auf Fragebögen, wie sie in früheren Studien verwendet wurden [4, 7]. Neben Informationen zur Anamnese und zu Behandlungsschema und -verlauf wurde nach der Stuhlfrequenz gefragt und die Ausprägung der obstipationsbedingten Beschwerden nach einer Skala von 0 bis $3(0=$ beschwerdefrei, $1=$ schwache, $2=$ mittlere, 3 = starke Ausprägung) bewertet. Insbesondere wurden die Symptome heftiges Pressen, Stuhlkonsistenz (knollig-hart oder weich-flüssig), Gefühl der inkompletten Entleerung, Notwendigkeit manueller Manöver zur Stuhlentleerung, Blähungen, Flatulenz, Bauchschmerzen und -krämpfe, Schmerzen beim Stuhlgang und Beeinträchtigung der täglichen Aktivität abgefragt. Weitere Fragen betrafen den Grund für die Wahl von Padma Lax anstatt anderer Behandlungsmöglichkeiten, relevante Laborbefunde, mögliche unerwünschte Wirkungen und Interaktionen, Vor- und Begleittherapien sowie eine Gesamtbeurteilung der Therapie durch den Arzt und den Patienten bezüglich Wirksamkeit, Verträglichkeit und Darreichungsform. Zur Verlaufsbeobachtung wurden diese Daten aus mindestens 2, maximal 4 Visiten erfasst. Die Resultate wurden gegengelesen und auf Konsistenz überprüft und die Daten gegebenenfalls nach Rücksprache mit dem Arzt korrigiert oder ergänzt.

Bei der Datenanalyse wurde zur Beurteilung des Verlaufs die letzte registrierte Visite berücksichtigt. In Anbetracht des deskriptiven Ansatzes wurde auf die Berechnung von Signifikanzen verzichtet.

Das untersuchte Präparat Padma Lax wird von der Padma AG in der Schweiz hergestellt. Eine Tablette enthält $35 \mathrm{mg}$ Alantwurzel, 12,5 mg auf 2,4-2,6 mg Hydroxyanthracenderivate (Auszugsmittel: Wasser) eingestellten Aloetrockenextrakt, 1,75 mg Brechnusssamen, 52,5 mg Cascararinde, $10 \mathrm{mg}$ Colombowurzel, $35 \mathrm{mg}$ Enzianwurzel, 52,5 mg Faulbaumrinde, 70 mg Ingwerwurzel, $10 \mathrm{mg}$ Kondurangorinde, 3,5 mg Langer Pfeffer, $35 \mathrm{mg}$ Myrobalanenfrucht, $15 \mathrm{mg}$ Natriumhydrogencarbonat, $35 \mathrm{mg}$ wasserfreies Natriumsulfat, $70 \mathrm{mg}$ Rhabarberwurzel und $25 \mathrm{mg}$ Weißer Ton.

\section{Ergebnisse}

Insgesamt nahmen 17 Ärzte, darunter 6 Allgemeinpraktiker, 3 Fachärzte der Inneren Medizin, 6 Gynäkologen sowie 1 Chirurg und 1 Spezialist für Psychosomatik an der Studie teil und erhoben die Behandlungsdaten von 174 Patienten. Dabei kamen rund $60 \%$ der Patienten aus der Allgemeinen und Inneren Medizin und rund 30\% aus der gynäkologischen Praxis. Bei den Patienten handelte es sich um 51 (29\%) Männer und $123(71 \%)$ Frauen. 8 Frauen waren schwanger. Das Altersspektrum war 18-100 Jahre (durchschnittlich $53 \pm 18$ Jahre), die Körpergröße betrug $168 \pm 9 \mathrm{~cm}$ und das Gewicht $71 \pm 13$ $\mathrm{kg}$ (Body Mass Index (BMI) $25 \pm 4 \mathrm{~kg} / \mathrm{m}^{2}$ ). 21 Patienten hatten eine ausgeprägte Adipositas mit einem BMI $>30 \mathrm{~kg} / \mathrm{m}^{2}$.

\section{Ursachen für die Obstipation}

Bei fast allen Patienten wurden mehrere Ursachen angegeben, bei $45 \%$ waren dies ausschließlich Lifestyle-Faktoren wie Mangel an körperlicher Betätigung, ungenügende Flüssigkeitsaufnahme, inadäquate Ernährung, psychischer Stress und Änderung der Lebensumstände. Bei 55\% wurden zusätzliche Faktoren genannt, darunter Grunderkrankungen wie Nervenschädigung (8), Uro-/Anogenitalprolaps (7), Inkontinenz (2) sowie Multiple Sklerose (MS), Colitis ulcerosa, Divertikulitis, rezidivierende Genitalinfekte, Fibromyalgie und Megacolon (je 1). Bei 43 Patienten lag ein IBS und bei 22 eine Slow-Transit-Obstipation vor. Bei 26 Patientinnen wurden hormonelle Ursachen angegeben, wobei 13 an Hyperandrogenämie, 11 an Hypothyreose und je 1 Patientin an polyzystischem Ovarialsyndrom bzw. Oligo-/Amenorrhoe litten. 8 Patientinnen waren schwanger und 3 in einer vorzeitigen Menopause. Weitere Ursachen waren Nikotinabusus (5), Adipositas (2), Operationen (2) und psychische Faktoren (1). Bei 20 Patienten wurde ein Abführmittelmissbrauch und bei 13 Arzneimittelnebenwirkungen als Mitursachen für die Obstipation angegeben. Die Evaluation der Ko-Medikation zeigte jedoch, dass insgesamt 41 Patienten Medikamente einnahmen, die häufig oder sehr häufig Verstopfung oder Symptome wie Bauchschmerzen und Blähungen als unerwünschte Wirkung verursachen. Darunter waren z.B. Analgetika (11) und Antidepressiva / selektive Serotonin-Wiederaufnahmehemmer (11) sowie Protonenpumpen-Inhibitoren (PPI) und Blutdrucksenker (je 6). Bei den Verstopfungsursachen konnten bei hormonellen Faktoren (nur Frauen), bei IBS (29\% Frauen > 16\% Männer) sowie bei neurogener Obstipation (Lähmungen) (14\% Männer $>1 \%$ Frauen) Geschlechtsunterschiede beobachtet werden.

\section{Gesamtbeurteilung Wirksamkeit}

Die Stuhlfrequenz stieg im Laufe der Behandlung mit Padma Lax deutlich an. Der Anteil mit einer Frequenz von $\leq 2$ pro Woche sank von 47 auf 3\% und jener mit täglichem Stuhlgang ( $\geq 7$ pro Woche) stieg von 1 auf $37 \%$ (Abb. 1a). Der Anteil an Patienten mit mittlerer oder starker Verstopfung sank von 88 auf $13 \%$, während bei $38 \%$ die Obstipation gänzlich verschwand (Abb. 1b). 86\% der Ärzte wie auch der Patienten beurteilten die Wirksamkeit als gut (Tab. 1). Bezüglich der Zufriedenheit mit der Behandlung erreichte die Therapie auf einer 10-cm-Skala $(0=$ sehr unzufrieden, $10=$ sehr zufrieden $) 8,1 \pm 1,7$. 
Tab. 1. Gesamtbeurteilung der Therapie mit Padma Lax bezüglich Wirksamkeit und Verträglichkeit durch den Arzt/Patienten

\begin{tabular}{|c|c|c|c|c|c|c|}
\hline & \multicolumn{2}{|l|}{ Gut } & \multicolumn{2}{|c|}{ Mittel } & \multicolumn{2}{|c|}{ Schlecht } \\
\hline & $\mathrm{n}$ & $\%$ & $\mathrm{n}$ & $\%$ & $\mathrm{n}$ & $\%$ \\
\hline Wirksamkeit & $149 / 149$ & $86 / 86$ & $19 / 22$ & $11 / 13$ & $5 / 3$ & $3 / 2$ \\
\hline Verträglichkeit & $145 / 138$ & $83 / 79$ & $28 / 33$ & $16 / 19$ & $1 / 3$ & $0,5 / 2$ \\
\hline
\end{tabular}

b

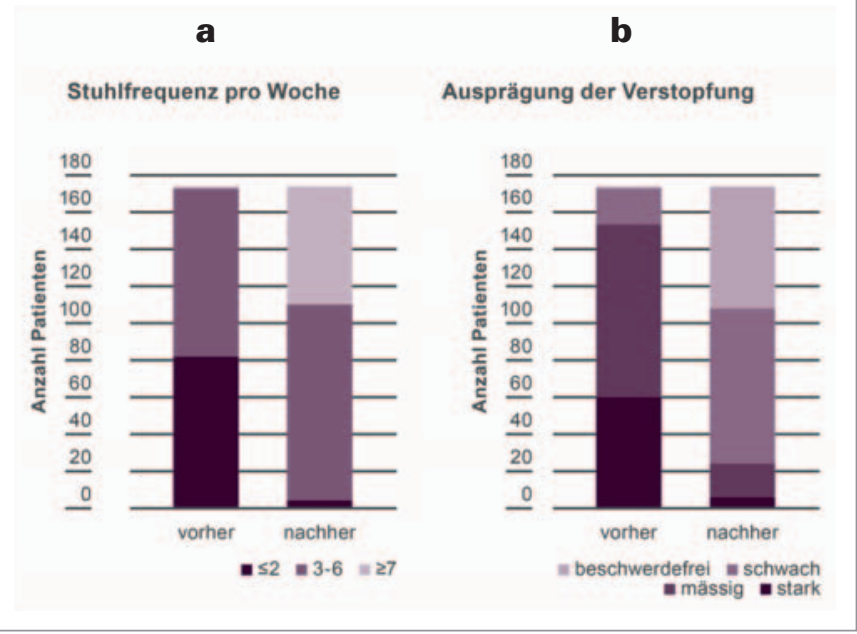

Abb. 1. Veränderung a der Stuhlfrequenz pro Woche und b der Ausprägung des Sympoms Verstopfung im Laufe der Padma-Lax-Behandlung $(\mathrm{n}=174)$.

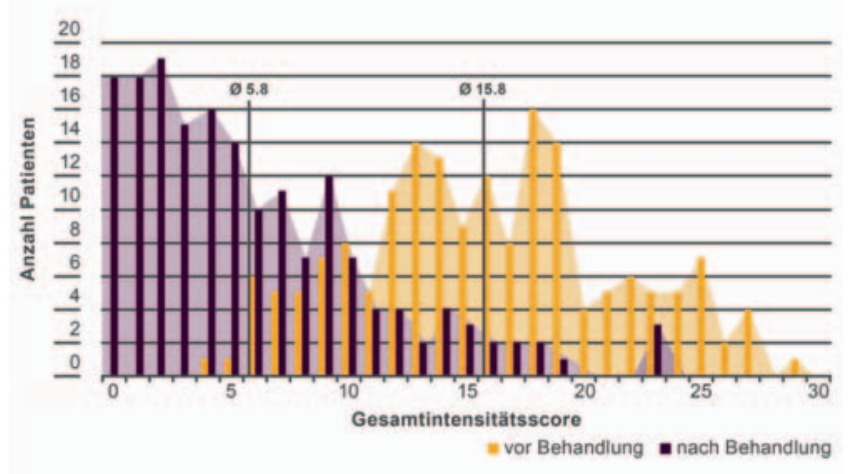

Abb. 2. Verteilung der Patienten nach Gesamtscore der Beschwerdeintensitäten aller Verstopfungssymptome vor und nach der Behandlung mit Padma Lax (Intensität: $3=$ stark, 2 = mäßig, 1 = schwach, $0=$ beschwerdefrei; maximal möglicher Score $=36$ ).

\section{Symptomspezifische Veränderungen}

Während anfangs fast alle Patienten an den Symptomen Verstopfung und knollig-harter Stuhl litten, wurden im Behandlungsverlauf rund $40 \%$ davon diesbezüglich beschwerdefrei. Weitere häufige Symptome waren mit etwa $90 \%$ der untersuchten Patienten heftiges Pressen, Blähungen und Flatulenzen. Davon wurden jeweils 51, 34 und 42\% beschwerdefrei. Bei den Symptomen Schmerzen beim Stuhlgang, Be- einträchtigung der täglichen Aktivität und Gefühl inkompletter Entleerung war der Anteil beschwerdefrei gewordener Patienten 67, 65 und 55\%, bei Bauchkrämpfen, Bauchschmerzen und der Notwendigkeit manueller Manöver waren es 53, 51 und $44 \%$. Einzig beim Symptom weicher/dünner Stuhl blieb die Häufigkeit mit $18 \%$ Betroffener vor und nach Behandlung gleich.

Die Intensität der Beschwerden wurde für jedes Symptom mit einer 4-Punkte-Skala angegeben ( 3 = stark, 2 = mäßig, $1=$ schwach und $0=$ beschwerdefrei). 97\% der Patienten zeigten eine Reduktion des Gesamtintensitätsscores, d.h. der Summe der Intensitätsangaben über alle 12 Symptome, von durchschnittlich 15,8 um 63\% auf 5,8. Der Anteil an Patienten mit hoher Gesamtintensität (Gesamtscore $>20$ ), d.h. mit vielen Symptomen von hoher Intensität, sank von 20,1 auf 1,7\%, jener der Patienten mit niedrigem Gesamtscore $(\leq 5)$ stieg von 1,2 auf $57 \%$. Insgesamt wurden über $10 \%$ völlig symptomlos (Abb. 2).

Die mittlere Intensität über alle Symptome sank um $63 \%$ von 1,84 auf 0,68. Die Anfangsintensität war bei Verstopfung und knollig-hartem Stuhl mit 2,2 am höchsten und reduzierte sich im Behandlungsverlauf um 64\% auf 0,8 (Abb. 3). Die Verminderung der Intensität war besonders deutlich bei der Beeinträchtigung der täglichen Aktivität und Schmerzen beim Stuhlgang mit einer Reduktion um $76 \%$, bei heftigem Pressen, Bauchschmerzen und dem Gefühl inkompletter Entleerung (Reduktion um 67\%). Die mittlere Intensität des Symptoms weicher/dünner Stuhl blieb mit 0,96 gleich.

\section{Vergleich von Untergruppen}

Die Patienten dieser Studie waren nicht nur bezüglich der demografischen Daten, sondern auch bezüglich der medizinischen Vorgeschichte sehr heterogen. Während kein Unterschied zwischen der Wirkung bei Männern und Frauen sowie bei älteren Patienten $\geq 70$ Jahre verglichen mit dem Durchschnitt beobachtet wurde, wichen die Resultate von Gruppen mit verschiedenen Obstipationsursachen zum Teil deutlich vom Durchschnitt ab (Abb. 4).

Mit einer Reduktion der Gesamtintensität der Obstipationsbeschwerden um $75 \%$ trat die stärkste Verbesserung bei durch Schwangerschaft verursachter Obstipation auf. Die Symptome weicher/dünner Stuhl und Notwendigkeit manueller Manöver kamen hier nicht vor, mit Ausnahme von Bauchschmerzen und -krämpfen waren aber alle anderen Symptome vor der Behandlung ausgeprägter als beim Patientendurchschnitt (Daten nicht abgebildet). Am deutlichsten war dies bei Flatulenzen und Blähungen, die bei den Schwangeren anfangs eine mittlere Intensität von 2,38 bzw. 2,75 hatten und die sich um $68 \%$ bzw. $77 \%$ auf 1,76 und 1,9 reduzierten.

Bei Patienten mit neurogener Obstipation, meist mit Paraoder Tetraplegie, traten vermutlich aufgrund der neurologischen Defizite keine Schmerzen beim Stuhlgang auf und das 
Abb. 3. Veränderung der mittleren Ausprägung der Symptome während der Behandlung mit Padma Lax $(\mathrm{n}=174)$.

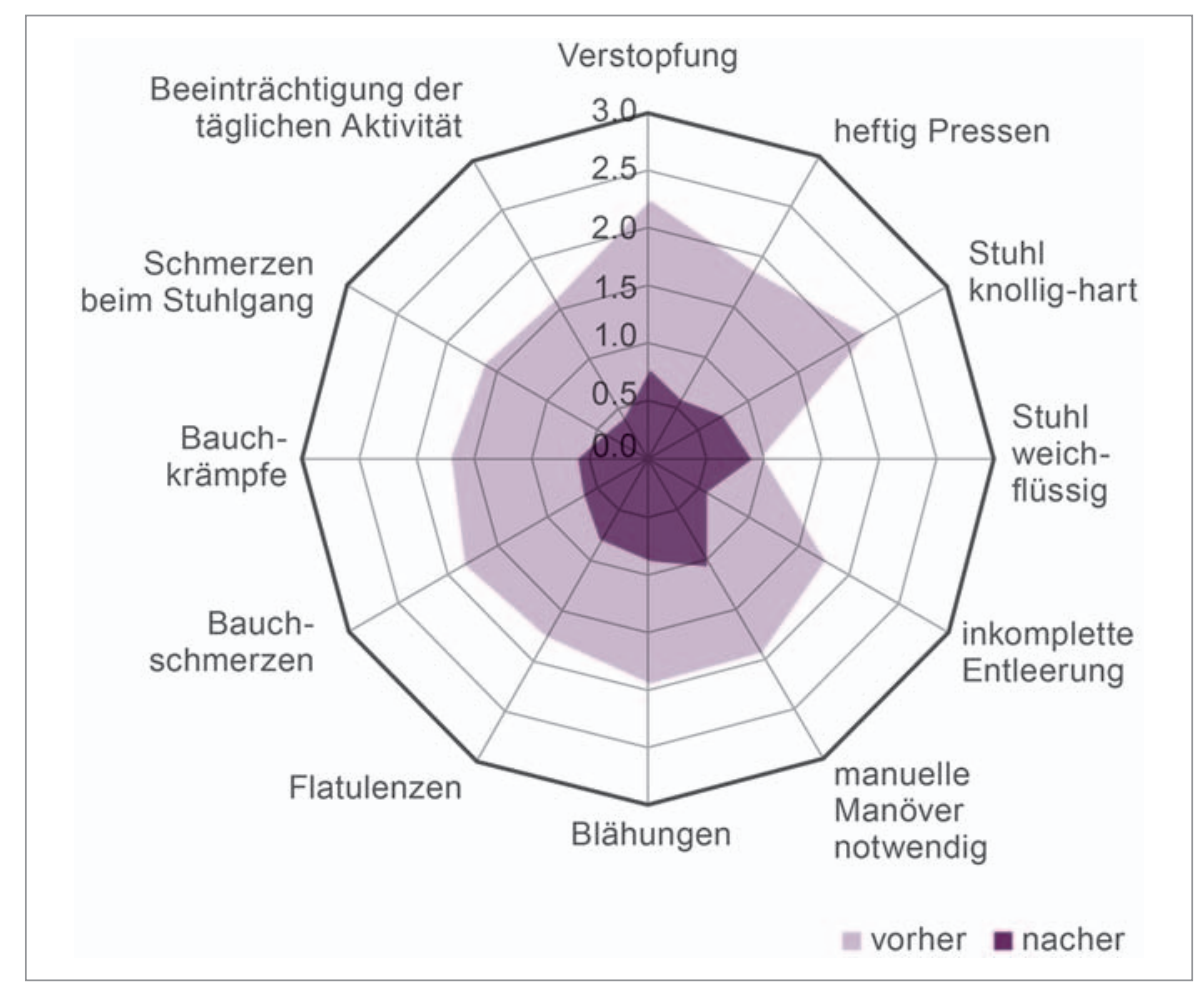

Abb. 4. Veränderung des Gesamtintensitätsscores über alle Obstipationssymptome durch Padma Lax bei verschiedenen Untergruppen.
Intensität der Obstipationssymptome vor und nach Behandlung mit Padma Lax

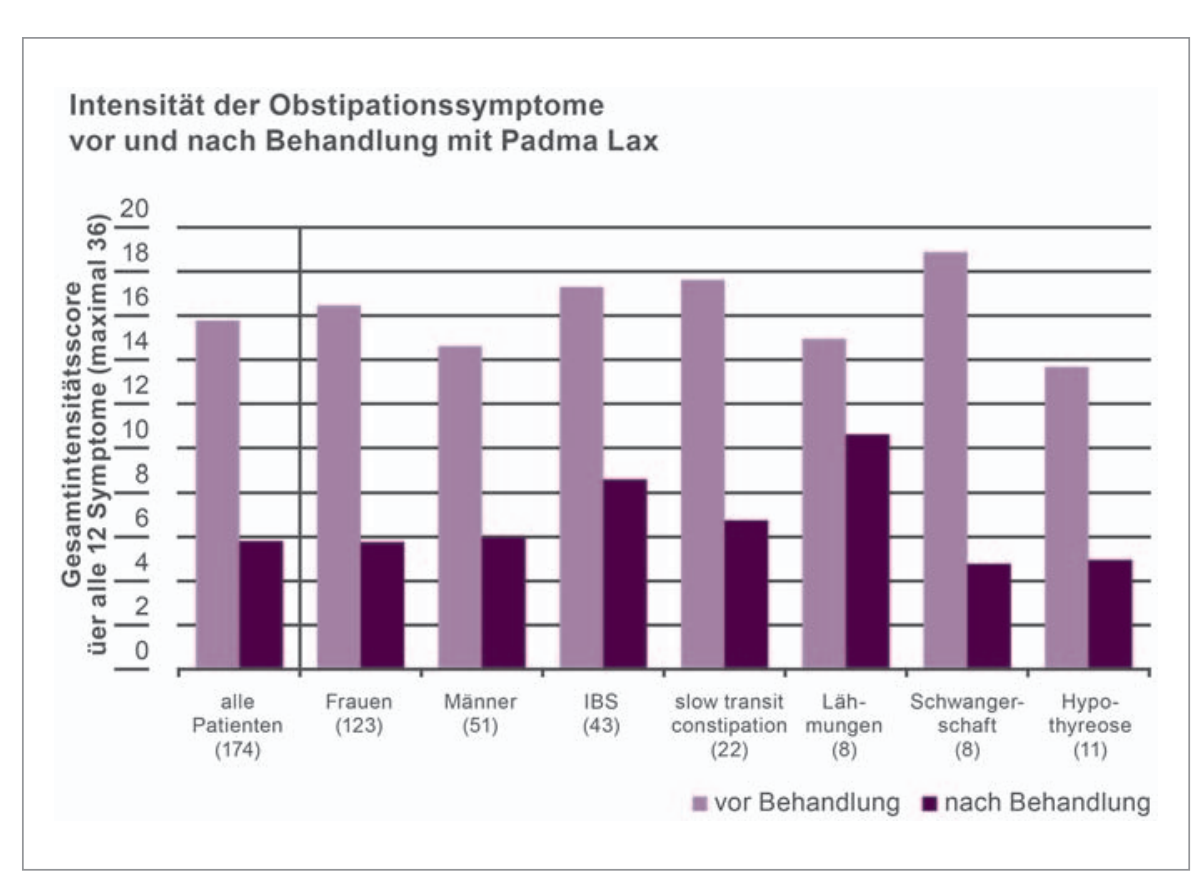

Stuhlkonsistenz weich/dünn

31 Patienten, darunter 14 mit IBS, litten zu Beginn sowohl an Verstopfung als auch an weichem/dünnem Stuhl. Bei 17 davon besserten sich beide Symptome, wobei 11 beschwerdefrei wurden. Bei 4 besserte sich nur die Verstopfung. 5 Patienten klagten über eine Verstärkung des dünnen Stuhls, während sich bei 4 Personen weder das eine noch das andere Symptom änderte. Bei 12 Patienten trat das Symptom während der Behandlung neu auf. 


\section{Dosierung und Therapiedauer}

Am häufigsten war die Dosierung 1-2 Tabletten (30 und $67 \%$ ). $75 \%$ nahmen das Präparat täglich, ansonsten war die Einnahmefrequenz alle 3-6 Tage oder nach Bedarf. Bei 34 Patienten wurde die Dosierung während der Therapie reduziert, in 22 Fällen aufgrund einer Besserung der Symptome und bei 6 Patienten aufgrund zu starker Wirkung bzw. zu weichem Stuhl. Bei 4 Patienten wurde die Dosierung erhöht, wobei bei 2 dieser Patienten bereits vor der Dosisänderung eine starke Besserung aufgetreten war (Gesamtintensitätsscore 22 auf 6 bzw. 26 auf 3). Mit einer Tagesdosis von 6,4 (je 2 Patienten) und 3 Tabletten nahmen 5 Patienten das Präparat anfangs in einer höheren als in der Packungsbeilage empfohlener Dosierung ein. Aufgrund einer Besserung der Beschwerden reduzierten alle die Dosierung im Lauf der Behandlung auf 1 oder 2 Tabletten täglich.

Die Therapiedauer bzw. bei fortgeführter Therapie die Beobachtungsdauer betrug im Median 2,35 Monate (7 Tage bis 13 Monate), wobei es sich im letzteren Fall um einen IBSPatienten handelte, bei dem die Behandlung unter der Dosierung 3 Tabletten pro Woche fortgeführt wurde. $48 \%$ der Patienten setzten die Behandlung mit Padma Lax nach dem Beobachtungszeitraum fort.

\section{Vor- oder Ko-Medikation}

Insgesamt waren 60 Patienten vor oder während der Therapie mit Padma Lax auch noch mit anderen Medikamenten gegen Obstipation behandelt worden. Dabei handelte es sich hauptsächlich um Quellstoffe (34\%), osmotische Laxantien (19\%) und Spasmolytika (14\%). Bei 34 Patienten führte die Behandlung mit Padma Lax zu einem Absetzen, bei 13 zum teilweise Absetzen oder einer Reduktion der Co-Medikation. Im Vergleich zu früheren Behandlungen gegen Obstipation erreichte die Therapie mit Padma Lax auf einer 10-cm-Skala $(0=$ viel schlechter, $10=$ viel besser, $5=$ gleich gut $)$ bei den Ärzten 7,8 $\pm 1,5$ und bei den Patienten 7,9 $\pm 1,7$.

\section{Therapieabbruch, Sicherheit und Verträglichkeit}

5 Patienten brachen die Therapie von sich aus vorzeitig ab. Grund dafür waren in je 1 Fall ein unerwünschtes Ereignis (UE), Unverträglichkeit, Therapieerfolg, Bevorzugung einer anderen (flüssigen) Galenik und Um-/Wegzug. Beim UE handelte es sich um Blähungen und Flatulenz, wobei beide Symptome bereits in mittlerer Intensität vorbestanden und sich die Flatulenz verstärkte. Es wurden nicht genügend Labordaten geliefert um Schlüsse zur Sicherheit zu ziehen. Die Verträglichkeit wurde von den Ärzten in $83 \%$ und von den Patienten in $79 \%$ der Fälle als gut beurteilt (Tab. 1).

\section{Diskussion}

Limitationen dieser Studie sind der retrospektive Ansatz mit der freien Auswahl von Patienten sowie die Verwendung eines nicht validierten Fragebogens. Es ist nicht auszuschließen, dass hierdurch eine Verzerrung zustande kommt, weil bestimmte Patienten mit besonders guter Response ausgesucht wurden. Bei multifaktoriellen Erkrankungen wie der Obstipation, bei denen auch psychische Faktoren an Entstehung und Verlauf beteiligt sind, spielt der Placeboeffekt eine wich- tige Rolle und kann nur durch entsprechende kontrollierte Studien erfasst werden. Die Beurteilung der Wirksamkeit bei einem Laxans hingegen wird recht gut durch die Stuhlfrequenz erfasst: Bei unmittelbar im zeitlichen Zusammenhang mit der Medikation auftretenden Änderungen dieses Parameters kann bei chronischer Verstopfung auf eine Kontrollgruppe verzichtet werden. Weitaus genauer und verlässlicher wäre aber anstelle globaler Angaben in der Patientenakte durch den Arzt ein entsprechendes Patiententagebuch.

Der retrospektive Studienansatz erlaubt jedoch eine Übersicht über die tatsächliche Anwendung des Präparats bezüglich Indikationen und Zielgruppen in der Praxis. Unsere Daten zeigen, dass Padma Lax bei einem breiten Patientenspektrum eingesetzt wird. Während $60 \%$ der Berichte aus der Allgemeinen und Inneren Medizin stammten, kamen rund $30 \%$ aus gynäkologischen Praxen, die anteilsmäßig zu 35\% vertreten waren. Darunter waren auch 8 Patientinnen mit Schwangerschaft als Obstipationsursache, eine im ersten Trimester (12. Woche), die anderen zwischen der 16. und 32. Woche. Obwohl die Aussagekraft wegen der kleinen Zahl begrenzt ist, ist dennoch bemerkenswert, dass sie anfangs den höchsten $(18,75)$ und am Behandlungsende den niedrigsten mittleren Gesamtintensitätsscore hatten $(4,75)$. Mit dieser Reduktion um 14 Punkte (75\%) konnte diese Untergruppe am meisten von der Behandlung mit Padma Lax profitieren.

Das Präparat wurde bei Patienten mit verschiedenen Grunderkrankungen eingesetzt. Obwohl auch ein kurzzeitiger Einsatz erfasst werden sollte, war der Median der Behandlungsdauer 2,35 Monate für ein Laxativum relativ lang. Die Daten weisen auf eine gute Verträglichkeit des Präparates hin, und eine Dosiserhöhung, die auf eine mögliche Gewöhnung hindeuten könnte, wurde nicht beobachtet. Als einziges gemeldetes UE wurde bei einem Patienten eine Verschlechterung der Flatulenz um 1 Punkt angegeben. Weil nur für die wenigsten Patienten Labordaten geliefert wurden, lassen sich keine Schlüsse über eventuelle Veränderungen der Laborwerte ziehen. Dazu liegen jedoch Daten aus einer Placebo-kontrollierten Studie mit 61 IBS-Patienten über 3 Monate vor [4], in der keine Veränderung der Laborwerte inklusive der Elektrolyte und keine Anzeichen einer Gewöhnung beobachtet wurden.

In der vorliegenden Untersuchung litten rund ein Viertel der Patienten an IBS, 15 davon offensichtlich unter einer Mischform mit gleichzeitig knollig-hartem und weichem/dünnem Stuhl. IBS-Patienten hatten durchschnittlich stärkere Beschwerden, und bei dieser Gruppe war die Reduktion der Symptome nicht ganz so ausgeprägt. Dennoch konnten auch sie von einer Reduktion des Gesamtintensitätsscores um 50\% profitieren. Dies spiegelt die Resultate einer klinischen Studie bei obstipationsdominantem IBS wider [4], die unter Padma-LaxTherapie eine signifikante Verbesserung der Verstopfung, des Schweregrads abdominaler Schmerzen, der Beeinträchtigung der täglichen Aktivität, der Blähungen und Flatulenz sowie der inkompletten Entleerung im Vergleich zu Placebo zeigten.

Mit Ausnahme des Symptoms weicher/dünner Stuhl verringerte sich bei allen abgefragten Symptomen die Häufigkeit und die mittlere Intensität. Die Häufigkeit reduzierte sich zwischen 34 (Blähungen) und 69\% (Schmerzen beim Stuhlgang). Die mittlere Intensität von anfangs 1,57 (Beeinträchtigung der täglichen Aktivität) bis 2,2 (Verstopfung) reduzierte sich auf $\leq 1$ (schwache Ausprägung). 
Beim Symptom weicher/dünner Stuhl änderten sich die absoluten Zahlen bezüglich Häufigkeit (18\%) und mittlerer Intensität $(0,95)$ nicht, jedoch änderte sich die Verteilung der Patienten. Bei 5 verstärkten sich die Beschwerden und bei 12 traten sie neu auf. Eine gewisse Verringerung der Stuhlkonsistenz ist bei einem Laxans in der Regel erwünscht und kann bei Erkrankungen, die eine erleichterte Defäkation erfordern oder bei denen Pressen vermieden werden sollte, z.B. postoperativ oder bei Hämorrhoiden, ein Hauptziel der Behandlung sein. Während bei den oben genannten Patienten die Stuhlkonsistenz zu weich wurde, trat bei ebenfalls 17 Patienten eine Verbesserung des Symptoms, d.h. eine Erhöhung der Konsistenz des anfangs zu weichen Stuhls auf. Dies weist darauf hin, dass Padma Lax nicht nur laxative, sondern auch regulative Wirkung auf die Verdauung und das Stuhlverhalten hat.

Diese regulativen Eigenschaften könnten darauf beruhen, dass in Padma Lax neben stimulierenden Laxantien auch osmotische, prokinetische, hydragoge und absorbierende Stoffe enthalten sind [8]. Weitere Pflanzenkomponenten haben spasmolytische, antidiarrhoische und säurebindende Eigenschaften; außerdem zählt eine ganze Reihe der Inhaltsstoffe von Padma Lax zu den Bittermitteln, die die Verdauung anregen und klassischerweise bei Magen-Darm-Beschwerden wie Völlegefühl oder Appetitlosigkeit eingesetzt werden [8, 9]. Dieser für Tibetische Rezepturen typische Vielstoffcharakter führt zu einer Multi-Target-Wirkungsweise, die im Fall von Padma Lax nicht nur abführend, sondern auch regulierend zu wirken scheint. Gleichzeitig sollen durch die geringe Dosierung der einzelnen Komponenten mögliche Nebenwirkungen minimiert werden [10]. Dementsprechend wurde die Verträglichkeit sowohl von den Ärzten als auch von den $\mathrm{Pa}$ tienten als sehr gut beurteilt.

\section{Schlussfolgerungen}

Die Resultate zeigen, dass Padma Lax bei Verstopfungssymptomen unterschiedlicher Ursache eingesetzt wird. Das Spektrum umfasste beide Geschlechter, junge und ältere bis sehr alte Patienten sowie Patienten mit unterschiedlichen Grunderkrankungen. Rund 30\% der Fälle stammten aus der gynäkologischen Praxis. Neben einer guten Wirksamkeit zeigte das Präparat auch eine hohe Sicherheit und Verträglichkeit. Die Wirkung bei unterschiedlichen Symptomen sowie die Wirkungsweisen der verschiedenen Inhaltsstoffe lassen auf einen Multi-Target-Wirkmechanismus schließen. Dies ist besonders günstig bei Erkrankungen mit chronischem Charakter und komplexer Ätiologie wie bei der chronischen Obstipation. Damit stellt Padma Lax eine Therapieoption bei Verstopfung unterschiedlicher Ursache dar, die einen Platz sowohl in der Allgemeinen und Inneren Medizin als auch in der gynäkologischen Praxis und in der Geriatrie hat.

\section{Dank}

Die Autoren danken den beteiligten Kolleginnen und Kollegen für die Erhebung der Daten.

\section{Disclosure Statement}

C.V. ist eine Mitarbeiterin der Padma AG. Ansonsten erklären die Autoren keine Interessenskonflikte.

\section{Literatur}

1 Kappler S: Chronische Obstipation - eine ständige Herausforderung: Behandlung des chronischen obstipierenden Reizdarm-Syndroms mit Padma Lax. Schweiz Z Ganzheitsmed 2008;20:205-206.

2 Feldhaus S: Behandlung der chronischen Obstipation eines tetraplegischen Patienten mit dem tibetischen Arzneimittel Padma Lax - ein Fallbericht. Forsch Komplementmed 2006;13(suppl 1):31-32.

3 Feldhaus S: Obstipation und Sigmadivertikulose: Therapie mit Padma Lax. Schweiz Z Ganzheitsmed 2005;17:200-203.

4 Sallon S, Ben-Arye E, Davidson R, Shapiro H, Ginsberg G, Ligumsky M: A novel treatment for constipation-predominant irritable bowel syndrome using Padma Lax, a Tibetan herbal formula. Digestion 2002;65:163-173.
5 Gschossmann JM, Krayer M, Flogerzi B, Balsiger BM: Effects of the Tibetan herbal formula Padma Lax on visceral nociception and contractility of longitudinal smooth muscle in a rat model. Neurogastroenterol Motil 2010;22:1036-1041.

6 Hofbauer S, Kainz V, Golser L, Klappacher M, Kiesslich T, Heidegger W, Krammer B, Hermann A, Weiger TM: Antiproliferative properties of Padma Lax and its components ginger and elecampane. Forsch Komplementmed 2006;13(suppl 1):18-22.

7 Rome Foundation: ROME III diagnostic criteria for functional gastrointestinal disorders. Appendix A. Rome Foundation 2012;885-897, www.romecriteria. org
8 Schilcher H, Kammerer S, Wegener T: Leitfaden Phytotherapie, ed 3. Jena, Elsevier, 2007.

9 Schulz V, Hänsel R: Rationale Phytotherapie Ratgeber für die ärztliche Praxis. Berlin, Springer, 1996.

10 Schwabl H, Vennos C, Saller R: Tibetische Rezepturen als pleiotrope Signaturen - Einsatz von Netzwerk-Arzneien bei Multimorbidität. Forsch Komplementmed 2013;20(suppl 2):35-40. 